Digital Curation and Online Resources: 3D Scanning of Surgical Instruments at the Royal College of Physicians and Surgeons of Glasgow for an Open University Learning Resource

Kirsty Earley, Paul Rea, Daniel Livingstone

Anatomy Facility, Thomson Building, School of Life Sciences, College of Medical, Veterinary and Life Sciences, University of Glasgow, Glasgow, United Kingdom

The School of Simulation and Visualisation, Glasgow School of Art, Glasgow, United Kingdom 
Miss Kirsty Earley (corresponding author)

5 Upland Road

Scotstoun

Glasgow

G14 9BG

Tel: 01415876485

Email: kirsty.earley@gmail.com

Dr. Paul Rea

University of Glasgow

University Avenue

Glasgow

G12 8QQ

Tel: 01413304366

Email: paul.rea@glasgow.ac.uk

Dr. Daniel Livingstone

School of Simulation and Visualisation

Pacific Quay 
Govan Road

Glasgow

G51 1EA

Tel: 01415661459

Email: d.livingstone@gsa.ac.uk 


\title{
Digital Curation and Online Resources: 3D Scanning of Surgical Tools at the Royal College of Physicians and Surgeons of Glasgow for an Open University Learning Resource
}

\begin{abstract}
Collection preservation is essential for the cultural status of any city. However, presenting them publicly risks damage. Recently this issue has been overcome by digital curation. Proposed is a method of digitisation using photogrammetry and virtual reality software. Items were selected from the Royal College of Physicians and Surgeons of Glasgow archives, and implemented into an online learning module for the Open University. Images were processed via Agisoft Photoscan, Autodesk Memento, and Garden Gnome Object 2VR. Although problems arose due to specularity, 2VR digital models were developed for online viewing. Future research must minimise the difficulty of digitising specular objects.
\end{abstract}

Keywords: digital curation, 3D scanning, photogrammetry, online resource. 


\section{Introduction}

\subsection{Introduction}

Historical collections are a cherished cultural resource, attracting tourists, inspiring minds, and educating generations. Museums play a major role in the culture of a city, which is often overlooked. The tiring work done by curators benefits not only the cultural status of a city, but society as a whole.

There are many hurdles that must be overcome by curators. The finances required to display collections publicly are often scarce, along with a limited space physically (Hein, 1998). This leaves only a minute percent of entire collections available to the public eye (Muller, 2002). Hence, it is understandable as to why museums are so often targeted for closure. They must evolve with society to survive (Adams, 2016; Ridge \& Birchall, 2015).

The level of public access is another matter that curators must consider. Glass cases are the standard mode of presentation for particularly delicate items. Such items are so fragile that lengths must be taken to preserve them. However, this in turn limits the public's experience of collections (McGregor, 2016). The challenge of safely displaying items of history is one that all curators experience, but currently there lacks a universal method that can be applied to all genres of collection (Barrett, 2014; Parkinson, 2015).

The use of modern technology to digitise collections is a common occurrence in curation (Khan, 2013). Digitisation allows curators to create digital models of artefacts, engaging with the technology-focused public, whilst safely preserving history (Proctor, 
2010). Not only does this give visitors the opportunity to browse collections before a visit, it also enables those who are physically unable to visit the museum to access full collections in both experience and information (Created Realities, 2002). The most popular mode of online presentation is via 2D images, however this reduces the level of reality, which can impinge on the learning experience (Jung, Choi, Lim, \& Leem, 2002). Collections can be better digitised by 3D scanning.

The financial cost and time required to 3D scan historical collections are very much dependent on the type of equipment used. Laser scanners can accurately measure the dimensions of target objects and convert this information into digital models. However, they are high-priced and out-with the financial possibilities for smaller museum institutions (Chaplin, 2014). Photogrammetry can be an effective substitute. This mode of 3D scanning involves photographing a target object in a 360-degree manner and aligning reference points between images to develop a model (McGlone, Mikhail, \& Bethel, 2004). Photogrammetry proves to be an inexpensive option for curators as all that is required is standard photography equipment.

Despite its ease of use, photogrammetry is not without its problems. The accuracy of photogrammetry is affected by the material of the target. The difficulty of scanning reflective items is a common theme in the field of photogrammetry (Nicolae, Nocerino, Menna, \&Remondino, 2014). The alignment of reference points can be distorted by the presence of reflected light on the object surfaces. Hence, digital models are incomplete and inaccurate. Unfortunately, solutions to the problems that specular items cause have yet to be discovered.

A disputed topic in education is the usefulness of online resources as learning tools (Hiltz \& Turoff, 2005). As online resources become more commonplace in the 
classroom, the quantity and quality of learning materials is of utmost importance. A variety of materials can be presented simultaneously via the internet, hence promoting a multi-sensory learning experience for a plethora of leaner types (Shams \& Seitz, 2008; Ally, 2008).

Historical collections are insights into the past that everyone can learn from, but are not always accessible. Hence, the creation of an online learning module featuring interactive versions of historical collections could connect audiences with history.

\subsection{Aims}

The aim of this study was to generate a group of digital models, (ideally 3D), of surgical tools via photogrammetry, and to implement these models into an online learning resource.

The sample group includes surgical tools held at the Royal College of Physicians and Surgeons of Glasgow (RCPSG) that made a significant impact on medicine during the Victorian era. The type of photogrammetry utilised will be close-range. To increase the likelihood of obtaining complete digital models, several software packages will be employed to process images- Agisoft Photoscan, Autodesk Memento, and Garden Gnome Object 2VR. It is hypothesised that the presence of reflected light from the specular surfaces will disrupt the development of 3D models.

Several learning materials will accompany the digital models in the online learning module, including text and 2D images. It will be aimed at those at the Open University (OU) studying "Medicine and Society in Europe, 1500-1930", with the hopes that the resource will not only benefit students, but also the general public. 


\section{Methods}

The stages of this study were twofold. Firstly, to 3D scan items of the RCPSG museum collection. Secondly, to implement the digital models developed into an online learning resource. This chapter gives insight into the set of surgical artefacts chosen, the form of 3D scanning employed, and the software packages used to create an interactive learning experience.

\subsection{Sample Group}

Six historical items were chosen specifically for their role in pioneering medicine; the Lister Carbolic Acid Spray, a set of Osteotomes, a Dental Key for tooth extraction, an Amputation Saw, a Liston Knife, and an X-ray Tube, (Figure 1).

Figure 1. Sample tools from the RCPSG collection. Starting from top left in a clockwise direction: i) Lister Carbolic Acid Spray, ii) X-ray Tube, iii) Dental Tooth Key, iv) Macewen's Osteotome, v) Liston Amputation Knife, vi) Amputation Saw.

All objects were composed of glossy materials, such as metal or glass. The presence of glare was suspected to prove problematic when matching up reference points during image processing.

\subsection{Data Collection}

\subsubsection{Photography Equipment}

The DSLR camera used in this study was a Nikon D5300. As image quality had a direct 
impact on digital model quality, several camera settings were compared to achieve optimum results.

The sample items could not be taken off-site, thus the lighting set up for photography was that of the settings in the lecture theatre allocated for photography. To ensure that glare levels were kept to a minimum, a combination of low light intensity and a $35 \mathrm{~mm}$ polarising lens filter was employed.

Static and dynamic photography were compared when capturing images. The camera remained stationary on a tripod stand during static photography, while the target object was rotated on a turntable by 10 degrees. Dynamic photography involved keeping the target object stationary while the camera was moved 10 degrees per image.

To retain all image details captured during photography, images were captured in RAW format.

\subsubsection{Photogrammetry}

The form of 3D scanning utilised in this study was photogrammetry. This technique processes 3D digital models via the alignment of reference points between images. 20-80 images were captured of each tool, taken from different angles to guarantee that the entire object was scanned. The scanning of objects in such a close proximity is characteristic of close-range photogrammetry (McGlone et al., 2004).

Once the tool was scanned in entirety, 3D digital models were processed and constructed on a variety of software programmes, described below. 


\subsection{Photogrammetric Software Packages}

The software packages used for image processing included Agisoft Photoscan and Autodesk Memento, and Garden Gnome Object 2VR. Due to the specularity of the surgical tools, it was expected that the packages would have difficulty processing the images and generating accurate 3D digital models. Glass reflects items in its surroundings- reflections change shape with every angle. Hence, the X-ray tube was not processed through Photoscan or Memento.

\subsubsection{Agisoft Photoscan}

Agisoft Photoscan is a popular programme in photogrammetry, building models from images to point clouds to meshes to the final textured model. For the purposes of this study, the Pro version was employed (Agisoft, 2016).

20 to 80 images were processed in Photoscan per session to guarantee full coverage of the object. Images of poor quality were excluded to ensure the proper alignment of reference points. Once developed, models were exported as OBJs.

\subsubsection{Autodesk Memento}

Another photogrammetry programme for image processing is Autodesk Memento (Autodesk, 2015). With a similar visualisation pipeline to Photoscan, Memento is more automatic, thus any occurring problems are difficult to resolve.

Similar to Photoscan, image input quantity affected the quality of the final textured 
model. Hence, 20-80 images were uploaded and processed via Memento. Once generated, models were exported as OBJs.

\subsubsection{Garden Gnome Object 2VR}

The final programme used was Garden Gnome Object 2VR. Unlike Photoscan and Memento, Object 2VR creates panoramic digital models, enabling the viewer to rotate the object across several planes (Garden Gnome, 2016). This is done by collating a series of images in such a fine sequence it mimics 3D movement (Figure 2). Hotspots were applied to the final $2 \mathrm{VR}$ models to provide more information on the tool, along with arrows for navigational control.

Figure 2: Example of image collation in Light Table application of Garden Gnome Object 2VR.

2VR models were exported as QT and HTML5 files. Final models from this study were output as manipulatable videos to enable full user control for viewers.

\subsubsection{D Modelling and Animations}

Attempts were made to manually develop 3D digital models via Autodesk 3DSMax. A 3D animation was designed to illustrate the use of the dental key during a tooth extraction. The online database Body Parts/Anatomography of Database Centre for Life Science (DBCLS), Tokyo, Japan, was the source of anatomical digital models used in the 3D animation (Database Centre for Life Science, 2009). Models were constructed on Unity and animated via 3DSMax. The animation was then rendered via Adobe After Effects. 


\subsection{Online Learning Module}

The structure of the online learning module was organised using interactive PDFs. Included in each PDF were several learning material types, such as text, 2D images and an interactive digital model.

The module was constructed via Moodle, a web based virtual learning environment (Dougiamas, 2016). The final version of the module was uploaded onto the RCPSG test website for reviewal before final release.

\section{Results}

After rounds of scanning, a complete set of interactive digital models was obtained. Models were implemented into an online learning module, which was aimed at students of the OU. Once scanned, photographs were processed by several computer software programmes and results compared.

\subsection{Photogrammetry}

Several rounds of photography were carried out to determine optimum conditions for image capture. This was necessary as the quality of the image had a direct impact on digital model quality.

The initial setting for image capture was the Automatic mode. This setting proved to produce a significant amount of noise, due to high ISO values (Figure 3). Hence, camera settings were altered to Manual to reduce the ISO value (Figure 4). 
Figure 3: Example of image obtained with Automatic camera settings. Note the high level of noise from high ISO.

Figure 4: Reduced level of noise in image obtained using Manual camera settings with low ISO.

To reduce the level of glare from the specular objects, a $35 \mathrm{~mm}$ polarising lens filter was applied. Although it did limit glare, the lens also limited the level of exposure and generated noise. However, it was possible to edit the images in Adobe Lightroom in order to overcome these barriers.

Specular objects not only distort reference points between images, but also image sharpness. Thus to ensure that the entire object was in focus, a high aperture ranging from f/14-18 was used along with a longer shutter speed to improve the level of exposure, and low ISO value to reduce noise. These settings were achieved using the Aperture-Priority mode, and generated the sharpest images (Figure 5). Due to the lengthened shutter speed, the tripod stand had to be administered, meaning that the average time required to scan all instruments was 8 hours.

Figure 5: Highest quality image achieved using Aperture-Priority settings.

\subsection{Digital Model Processing}

Different software packages were compared when processing digital images. The outcomes of image processing are discussed below.

\subsubsection{Agisoft Photoscan}

As has been discussed previously, the presence of glare produced by specular objects causes problems when attempting to align images (Nicolae et al., 2014; Reich, 
Ritter, \&Thesing, 2000). The same issues occurred in the present study, with Photoscan having difficulty in matching reference points between images.

Initially, the time required to produce a model in Photoscan was 15 minutes. However, when the number of images to be processed increased to 80 per object, the average processing time was 2 hours.

When comparing the effect of camera settings on digital model quality, it is clear that models became more accurate as settings were altered from Automatic to AperturePriority. Although the Automatic settings made photographing relatively easy, the resulting digital models were very distorted; the high level of noise and glare malformed surfaces and textures, with many of the object components missing (Figure 6).

Figure 6: Incomplete textured model in Photoscan. Note the presence of noise and absence of tool components.

Model quality improved notably when the polarising lens filter was applied. Specularity was significantly reduced, producing more complete textured models. Despite the improvements, models were still incomplete and not of a high enough quality to be included in the learning module (Figure 7).

Figure 7: Example of final textured model of best quality using Agisoft Photoscan.

The alignment of cameras proved to be problematic. Any presence of glare disrupts the matching of reference points between images. Thus, regardless of the number of photographs captured, Photoscan always had difficulty with alignment. To improve this, target stickers were placed around the artefact to act as reference points them- 
selves. This use of "artificial reference points" allowed for better camera alignment, rendering more accurate digital models (Figure 8, Figure 9).

Figure 8: Alignment of camera angles without reference stickers.

Figure 9: Alignment of camera angles with reference stickers.

Another comparison made was the efficacy of static and dynamic photography. It was found that static photography resulted in more precise reference point alignment, which lead to higher quality digital models.

Overall, the most accurate digital models were produced using static photography, accompanied with the Aperture-Priority mode, a polarising lens filter, and target stickers.

Granted that many attempts were made to render complete 3D digital models via Photoscan, the presence of glare proved to be too strong a barrier to overcome. Hence, Photoscan models were not included in the final online learning module.

\subsubsection{Autodesk Memento}

The second software programme utilised in this study was Autodesk Memento. Memento requires less user input than Photoscan, allowing little control over the visualisation pipeline. Thus, no comparison could be made between reference point alignment in Photoscan and Memento.

Similar results in model quality were produced in Memento as with Photoscanthe final models were highly distorted and incomplete. The presence of reflected light again interfered with image alignment. Although alignment and model quality did im- 
prove after the placement of reference stickers, highly specular areas were not well processed (Figure 10).

Figure 10: Final rendered model of Lister Carbolic Acid Spray via Autodesk Memento.

Memento proved to be more problematic than Photoscan. Although the visualisation pipeline was more automatic, textured models were hard to obtain- entire projects were lost due to the programme crashing. These issues have no obvious solution as little research has been performed with Memento (Thimmesch, 2015).

\subsubsection{Garden Gnome Object 2VR}

Unlike the photogrammetric programmes, Object $2 \mathrm{VR}$ is not affected by the presence of glare, and does not generate models by reference point alignment. Instead, it collates the images in such a fine sequence that manipulation of the model mimics 3D movement. Thus, 6 interactive 2VR models were generated using Object 2VR. These models enable the user to view the object in a 360-degree fashion (Figure11).

Figure 11: Example of final Object 2VR model of X-ray Tube.

The images were edited in Adobe Photoshop to present the tools with different background options. The final aesthetic chosen was a simple white background, which placed all focus on the tool itself (Figure 12).

Figure 12: Photoshop background editing. 
Hotspots were applied to provide further details on the surgical tools (Figure 13). These labels appeared when hovering the mouse over different tool components (Garden Gnome, 2015). This technique helped to signify the importance of each component.

Figure 13: Object 2VR model with hotspots.

Due to its immunity to glare and reflected light, Object 2VR is an effective alternative to Photoscan or Memento. The quick processing time, lack of restrictions with regards to object materials, and simple layout makes $2 \mathrm{VR}$ a fail-safe digitisation tool.

\subsubsection{Manual Digital Modelling}

As a final attempt to develop 3D models, distorted models were retopologised in $\mathrm{Au}-$ todesk 3DSMax. This technique generates a smoother surface on top of the original distorted model (Figure 14, Figure 15).

Figure 14: OBJ import to Autodesk 3DSMax from Agisoft Photoscan.

Figure 15: process of retopology in 3DS Max.

As can be expected, retopology is a long process- on average, the retopology of one model took up to 8 hours. This was because of the manual approach taken to generate more accurate surfaces. Due to a limited time period, this mode of modelling was discarded.

Although 3D digital models could not be designed using 3DS Max, it was possible to create a 3D animation of the mechanism of the dental key during a tooth extrac- 
tion. This was done to include a 3D digital learning material in the online module. The dental key was modelled and animated using 3DS Max, with scenes being rendered on Adobe After Effects. Despite being a lengthy process, the animation was a favoured addition to the module.

\subsection{Module Development}

The conclusion of the study was the production of an online learning resource for the OU course "Medicine and Society in Europe, 1500s-1930s".

As the models generated by Photoscan and Memento were highly inaccurate, the Object 2VR models were the only products included in the learning resource. They were uploaded to the module as QT and HTML files.

Webpages of the module were initially organised on interactive PDFs via Adobe InDesign (Figure 16). Each page contained a variety of learning materials; information on the tool and its uses, 2D images from original articles, and a 2VR digital model. The home page detailed learning outcomes of the module, as well as how to navigate through the resource. The sequence of pages was in chronological order to reflect the age of each surgical tool. The module then concluded with a quiz (Figure 17). Once the structure was finalised, all of the materials were transferred from the PDFs to the module on Moodle (Figure 18).

Figure 16: Example of interactive PDF for William Macewen's Osteotome.

Figure 17: Module quiz format.

Figure 18: Home page of RCPSG online learning module. 
When creating an online learning resource, it is important to keep in mind the intended target audience (Ally, 2008). Hence, it was ensured that all content and learning materials were relevant for those studying at the Open University.

\section{Discussion}

\subsection{Introduction}

The presentation of historical items is a difficult task when the objects themselves are so fragile. The implementation of digital technology into museum exhibitions safely allows curators flexibility in how they display historical collections. Combined with useful learning materials, digitisation enables museum institutions to present academic resources online and make entire collections accessible worldwide.

It was the aim of this project to digitise a group of surgical instruments from the archives of the RCPSG for presentation in an online learning module. The tools in the sample group dated from the late 1700s-1800s, a time of significant advancement in medicine. Hence, their backgrounds were very relevant to those studying "Medicine and Society in Europe, 1500s-1930s" at the OU. The mode of digitisation was photogrammetry, with final digital models being developed via Garden Gnome Object 2VR. The digital models were then included in an online resource made available on the RCPSG Moodle website. It was hypothesised that the production of accurate 3D digital models would be unfeasible due to the specularity of the artefacts. As Photoscan and Memento rely on the alignment of reference points between images, the visualisation pipelines of these packages were disturbed, leading to incomplete 3D models. Such findings have already been noted in previous research. Thus, the digital models presented online were 
in fact 2VR models and not 3D.

Although museums present history, it is essential that they remain connected and relevant to the present times (Khan, 2013). With financial cuts being an ever-present threat, they must keep up to date with the changing culture in order to attract visitors. A way to do so is to adopt popular modes of communication, such as social media. The use of such technology connects museum institutions to the world, providing an accessible platform for their historical collections (Museums Association, 2005). Museums play a significant role in the cultural status of a city, reminding the public of history to impact the future. It is therefore crucial that these institutions are kept in existence.

\subsection{D Scanning and Photogrammetry}

The digitisation of entire historical collections can be an expensive option that curators often rule out. A solution to potential financial limitations is the use of close-range photogrammetry to digitise artefacts. However, this form of 3D scanning is not applicable to every artefact type.

Within this study, all artefacts were surgical tools composed of metal and/or glass. Such specular materials affect the sharpness of captured images, and ultimately the identification of common reference points when processing said images (Nicolae et al., 2014). This disruption in image alignment generates incomplete digital models that do not accurately reflect the original. Although attempts were made in this study to reduce the level of glare by way of dimmer lighting, reflected light was still an ever-present problem. 
Overcoming the barrier of specularity is a common occurrence in the field of photogrammetry, with many scholars proposing different solutions (Reich et al., 2000; Herbort et al., 2013). High quality 3D digital models of specular artefacts have been obtained by the application of more expensive equipment than that described in this study. The use of multiple cameras and polarising filters makes it possible to better control and reduce the level of reflected light (Wells, Jones, \& Danehy, 2005). A more invasive method of glare reduction is the application of matte powder to the artefact surface, producing complete digital models (James et al., 2015). Yet, whether this technique would be appropriate when digitising historical artefacts is questionable. Hence, although an effective approach, matte powder was not used in the present study.

Whilst the equipment did restrict the digitisation of the surgical tools, there were ways to overcome this. It was possible to decrease the level of specularity by applying a $35 \mathrm{~mm}$ polarising lens filter to the camera. This improved image alignment. Image alignment was further improved when stickers were placed around the target object. Combining these methods with the alteration of images in Adobe Lightroom, rendered models gradually became more intact. A minor detail, but nonetheless a triumph.

In summary, the presence of glare disrupted the rendering of complete 3D digital models of the surgical tools. Due to their poor quality, the digital models rendered by Photoscan and Memento were not included in the final module. To ensure that photogrammetry is an effective mode of digital curation, more research is needed to resolve the problems that specularity causes.

\subsection{D Virtual Reality Models}

Reflected light had no detrimental effects on image processing via Garden Gnome Ob- 
ject $2 \mathrm{VR}$. The visualisation pipeline of Object $2 \mathrm{VR}$ involves the sequencing of images in order to mimic 3D movement, hence is not reliant on the alignment of reference points (Garden Gnome, 2016). Thus, it was possible to render a complete set of 2VR digital models.

Ideally, 3D digital models would have been implemented in the learning module. Although panoramic movies enable 360 degree viewing of an object, they are not strictly $3 \mathrm{D}$. Future research is required to determine the efficacy of such models in creating a realistic and interactive learning experience.

A disadvantage of processing models via Object $2 \mathrm{VR}$ is the limited output files available. 2VR models are only available as QuickTime (QT), HTML5 or Flash files, which are not compatible with all internet browsers. Furthermore, Flash files are due to be phased out in the near future, making digital models in a Flash format would be inaccessible (Jobs, 2010). This would limit the accessibility of the learning module. It is essential that students can access the module on an anytime-anywhere basis.

Medical visualisation techniques are still relatively novel. Most research thus far has focused on the 3D scanning of cadaveric subjects for educational resources (Jocks, Livingstone, \& Rea, 2015; McMenamin, Quayle, McHenry, \& Adams, 2014). Few scholars have investigated the digitisation of medical artefacts and, thus far, none have applied close-range photogrammetry. Thus, the results of this study are one of a kind.

Despite the fact that the digital models included in the learning module were not $3 \mathrm{D}$, they were fully interactive. $2 \mathrm{VR}$ models can be manipulated in such a manner that users can view the object from a variety of angles. It is hoped that the results of this study can prove that close-range photogrammetry combined with Object $2 \mathrm{VR}$ is an inexpensive and effective method of digital curation. 


\subsection{Development of Online Learning Resource}

Once digitisation was complete, a learning resource for those studying at the OU was created and made available online via Moodle. The module was designed around the learning objectives of the medical history course.

Previous studies have highlighted the benefits of using 3D learning tools in academic subjects, improving understanding and concentration (Guidi, Barsanti, Micoli, \& Russo, 2015). Although it was not possible to generate 3D digital models in this study, the final products were interactive. Whether 2VR digital models, or any other form of 2D model, are as effective as 3D models is yet to be discovered. In fact, there is no consistent evidence to prove that 3D learning materials will always trump 2D materials. Instead, a combination of both $2 \mathrm{D}$ and $3 \mathrm{D}$ visual models has proven to be more advantageous than limiting resources to one type (Peterson, 2014).

The ability to interact with $2 \mathrm{VR}$ models immerses the user in a more realistic learning experience than simply presenting 2D images. With regards to digital curation, VR technology has not been utilised by museums institutions to a great extent. Thus, the products of this study are some of the few examples of VR technology in museum curation in the world.

The use of the internet is becoming a necessary area of museum management (Padilla-Melendez, \& del Aguila-Obra, 2013). A plethora of learning materials were incorporated into the online learning module along with 2VR models. This practice is known as active learning, which aims to connect to several learner types simultaneously (Bonwell \& Eison, 1991). A drawback of this is the risk of cognitive overload. Present- 
ing a variety of learning tools at once can activate several senses concurrently, which could confuse learners and impede on their learning experience (Mayer \& Moreno, 2003). Therefore, it was ensured that webpages of the learning module were structured in a simple and comprehensible manner.

\subsection{Limitations and Further Research}

A number of research projects can be proposed from the results and limitations of this study.

An alternative approach to digitising surgical artefacts would be to use a different photography set up. The control of light intensity was limited in this project by the fact that the tools could not be taken off-site. Thus, better digitisation results could be gained by investing in additional lighting and photography equipment.

As there is a wide variety of 3D scanning technology, it would be useful to investigate and compare different modalities. This could involve comparing the accuracy and ease of using close-range photogrammetry to using laser scanners (Evin et al., 2016). However, this may not be as much of a concern to museum curators with a limited funding budget (Hein, 1998).

The main hurdle with regards to digitising surgical artefacts is the presence of glare (Wang \& Feng, 2016). Time and again glare has resulted in inaccurate digital model representations, and the results of this study are no different (Nicolae et al., 2014; Guidi et al., 2015). Because the surgical tools were so fragile, only non-invasive methods of glare reduction could be taken. More invasive approaches increase the likelihood of generating complete 3D digital models (James et al., 2015). However, such methods cannot reasonably be applied universally. A potential solution would be to combine 
close-range photogrammetry with 3D modelling to modify incomplete models. There are also attempts being made to generate digitisation algorithms to improve model processing (Herbort et al., 2013). More research is required to determine a digitisation technique that can produce high quality results whilst complying to ethical standards.

There are many future plans to continue the work of this project and attempt to address questions that could not be answered in the allotted time. An extension of digital collections at the RCPSG is currently in place, with the hopes that the entire museum collection can be displayed online (McGregor, 2016). There are also plans to obtain feedback on the learning module from both students and members of the public- this was not possible due to time restrictions. Feedback on the structure and content of the learning resource would be useful to determine whether such online tools are beneficial in knowledge retainment. However, the debate on learning material efficacy is complex, and cannot solely be resolved by one factor (Tait, 2015).

\section{Conclusion}

In summation, an interactive online learning module focusing on a sample of the RCPSG museum collection was able to be developed. This was possible through the application of close-range photogrammetry and Object 2VR.

Displaying collections online creates an equilibrium between artefact preservation and presentation, whilst also ensuring that museums have an active presence in the digital world. Although it is necessary to contain items in glass cabinets, they do not have to be restrained. Online learning resources detailing artefacts from history can break down the barriers of traditional curation, and reach out to audiences worldwide. 
Thus, it is hoped that the results of this study can bring museums ever closer to an equal balance of preservation and presentation.

\section{Acknowledgements}

The authors would like to thank staff at the Library and Heritage department of the Royal College of Physicians and Surgeons of Glasgow, especially Carol Parry, Ross McGregor and Stuart Milligan. Thanks must also be given to medical scholars throughout history who worked tirelessly to advance clinical practice. Their research was truly revolutionary.

Word count: 4,881 . 


\section{References}

Adams, G.K. (2016, July 6). Museum across the UK face closure threat. Museums Association News. Retrieved from: https://www.museumsassociation.org/museums-journal/news/06072016-axe-to-museum-services

Agisoft. (2016). Agisoft Photoscan (Version 1.2.4) [Computer software]. St Petersburg, Russia: Agisoft LLC.

Ally, M. (2008). Foundations of educational theory for online learning. In T., Anderson (Ed), The Theory and Practice of Online Learning (15-44). Alberta: Athabasca University Press.

Autodesk. (2015). Autodesk Memento (Version beta) [Computer software]. San Rafael, CA: Autodesk, Inc.

Barrett, K. (2014, August). Preservation vs. Presentation: is digital display a solution for museums? Apollo Magazine. Retrieved from: http://www.apollo-magazine.com/ conservation-vs-access-digital-displays-solution-museums/

Bonwell, C.C., \& Eison, J.A. (1991). Active Learning: Creating Excitement in the Classroom. ERIC:Washington.

Chaplin, S. (2014). The medical library is history. RBM: A Journal of Rare Books, Manuscripts, and Cultural Heritage, 15, 146-156. Retrieved from: http://rbm.acrl.org/content/15/2/146.extract

Created Realities. (2002). The future of virtual museums: online, immersive, 3D environments. Texas: Jones, G. \& Christal, M.

Database Centre for Life Science. (2009). Body Parts 3D/Anatomography. Retrieved from: http://lifesciencedb.jp/bp3d/

Dougiamas, M. (2016). Moodle (Version 3.1.2.) [Computer software]. Perth, Australia: Moodle Pty Ltd.

Evin, A., Souter, T., Hulme-Beaman, A., Ameen, C., Allen, R., Viacava, P., Larson, G., Cucchi, T., \& Dobney, K. (2016). The use of close-range photogrammetry in zooarchaeology: creating accurate 3D models of wolf crania to study dog do- 
mestication. Journal of Archaeological Science: Reports, 9, 87-93. doi: http:// dx.doi.org/10.1016/j.jasrep.2016.06.028.

Garden Gnome. (2016). Garden Gnome Object 2VR (Version 3.1.1.) [Computer software]. Vienna, Austria: Garden Gnome Software e.U.

Guidi, G., Barsanti, S.G., Micoli, L.L., \& Russo, M. (2015). Massive 3D digitization of museum contents. In Toniolo, L., Boriani, M., \& Guidi, G., (Eds.), Built Heritage: Monitoring Conservation Management (335-346). Gewerstrasse: Springer.

Hein, G.E. (1998). Learning in the museum. Oxon:Routledge.

Herbort, S., Gerken, B., Schugk, D., \& Wohler, C. (2013). 3D range scan enhancement using image-based methods. ISPRS Journal of Photogrammetry and Remote Sensing, 84, 69-84. doi: http://dx.doi.org/10.1016/j.isprsjprs.2013.07.004.

Hiltz, S.R., \& Turoff, M. (2005). Education goes digital: the evolution of online learning and the revolution in higher education. Communications of the Association of Computing Machinery, 48, 59-64. doi: 10.1145/1089107.1089139.

James, D.W., Eckermann, J., \& Belblidia, F. (2015). Point cloud data from Photogrammetry techniques to generate 3D Geometry. In: Association for Computational Mechanics in Engineering. Proceedings of the 23rd UK Conference of the Association for Computational Mechanics in Engineering. Swansea: ACME. doi: 10.1061/(ASCE)SU.1943-5428.0000097.

Jobs, S. (2010, April). Re:Thoughts on flash. Retrieved from: http://www.apple.com/ hotnews/thoughts-on-flash/.

Jocks, I., Livingstone, D., \& Rea, P. (2015). An investigation to examine the most appropriate methodology to capture historical and modern preserved anatomical specimens for use in the digital age to improve access: a pilot study. Presented at the 9th International Technology, Education and Development Conference, Madrid, Spain. Retrieved from: http://eprints.gla.ac.uk/103652/

Jung, I., Choi, S., Lim, C., \& Leem J. (2002). Effects of Different Types of Interaction on Learning Achievement, Satisfaction and Participation in Web-Based Instruc- 
tion. Innovations in Education and Teaching International, 39, 153-162. doi: http://dx.doi.org/10.1080/14703290252934603

Yasmin Khan. (2013, January 22). Re: Museums in the information age: survival of the most digital? Retrieved from: http://www.collectionstrust.org.uk/blog/past-posts/ item/1603-museums-in-the-information-age-survival-of-the-most-digital

Mayer, R.E., \& Moreno, R. (2003). Nine ways to reduce cognitive load in multimedia learning. Educational Psychologist, 38, 43-52. doi: http://dx.doi.org/10.1207/ S15326985EP3801_6

McGlone, J.C., Mikhail, E.M., \& Bethel, J.S. (2004). Manual of Photogrammetry. Pennsylvania:American Society for Photogrammetry and Remote Sensing.

Ross McGregor. (2016, June 23). Re: Uncovering our Medical Instruments. Retrieved from: https://libraryblog.rcpsg.ac.uk/2016/06/23/uncovering-our-medical-instruments/

McMenamin, P.G., Quayle, M.R., McHenry, C.R., \& Adams, J.W. (2014). The production of anatomical teaching resources using three-dimensional (3D) printing technology. Anatomical Sciences Education, 7, 479-486. doi: 10.1002/ase.1475.

Padilla-Melendez, A., \& del Aguila-Obra, A.R. (2013). Web and social media usage by museums: online value creation. International Journal of Information Management, 33, 892-898. doi: http://dx.doi.org/10.1016/j.ijinfomgt.2013.07.004

Müller, K. (2002). Museums and Virtuality. Curator: The Museum Journal, 25, 21-33. doi: 10.1111/j.2151-6952.2002.tb00047.x.

Museums Association. (2005). Collections for the future. London: Museums Association.

Nicolae, C., Nocerino, E., Menna, F., \& Remondino, F. (2014). Photogrammetry applied to problematic artefacts. International Archives of the Photogrammetry, Remote Sensing and Spatial Information Sciences, XL, 451-456. doi: http://dx.doi.org/ 10.5194/isprsarchives-XL-5-451-2014. 
Parkinson, J. (2015, July 22). What makes a great museum? BBC News. Retrieved from: http://www.bbc.co.uk/news/magazine-33184094

Peterson, D. (2014). Quantitative and qualitative examination of 2D versus 3D technologies in teaching anatomy. The FASEB Journal, 28, 211.7. Retrieved from: http://www.fasebj.org/content/28/1_Supplement/211.7.

Proctor, N. (2010). Digital: museum as platform, curator as champion, in the age of social media. Curator: The Museum Journal, 53, 35-48. doi: 10.1111/j. 2151-6952.2009.00006.x.

Reich, C., Ritter, R., \& Thesing, J. (2000). 3D shape measurement of complex objects by combining photogrammetry and fringe projection. Optical Engineering, 39, 224-231. doi: 10.1117/1.602356.

Ridge, M., \& Birchall, D. (2015, October 25). How digital tech can bridge gaps between museums and audiences. The Guardian. Retrieved from: https://www.theguardian.com/culture-professionals-network/2015/oct/23/digital-technologymuseums-audiences-collaboration

Shams, L., \& Seitz, A. (2008). Benefits of multisensory learning. Trends in Cognitive Sciences, 12, 411-417. doi: 10.1016/j.tics.2008.07.006.

Tait, P. (2015, June 17). Intelligence cannot be defined by exams. The Guardian. Retrieved from: http://www.telegraph.co.uk/education/educationopinion/ 11678216/Intelligence-cannot-be-defined-by-exams.html

Thimmesch, D. (2015, February 26). Re: Autodesk releases free public beta version of its reality capture software, Memento. Retrieved from: http://3dprint.com/47082/ autodesk-public-beta-memento/

Wang, Y., \& Feng, H.Y. (2016). Effects of scanning orientation on outlier formation in 3D laser scanning of reflective surfaces. Optics and Lasers in Engineering, 81, 35-45. doi: http://dx.doi.org/10.1016/j.optlaseng.2016.01.003.

Wells, J.M., Jones, T.W., \& Danehy, P.M. (2005). Polarization and color filtering applied to enhance photogrammetric measurements of reflective surfaces. Paper 
presented at the 46TH AIAA/ASME/ASCE/AHS/ASC Structures, Structural Dynamics and Materials Conference, Texas.

\section{Figure Caption List}

Figure 1. Sample tools from the RCPSG collection. Starting from top left in a clockwise direction: i) Lister Carbolic Acid Spray, ii) X-ray Tube, iii) Dental Tooth Key, iv) Macewen's Osteotome, v) Liston Amputation Knife, vi) Amputation Saw.

Figure 2: Example of image collation in Light Table application of Garden Gnome Object 2VR.

Figure 3: Example of image obtained with Automatic camera settings. Note the high level of noise from high ISO.

Figure 4: Reduced level of noise in image obtained using Manual camera settings with low ISO.

Figure 5: Highest quality image achieved using Aperture-Priority settings.

Figure 6: Incomplete textured model in Photoscan. Note the presence of noise and absence of tool components.

Figure 7: Example of final textured model of best quality using Agisoft Photoscan.

Figure 8: Alignment of camera angles without reference stickers.

Figure 9: Alignment of camera angles with reference stickers.

Figure 10: Final rendered model of Lister Carbolic Acid Spray via Autodesk Memento.

Figure 11: Example of final Object 2VR model of X-ray Tube.

Figure 12: Photoshop background editing. 
Figure 13: Object 2VR model with hotspots.

Figure 14: OBJ import to Autodesk 3DSMax from Agisoft Photoscan.

Figure 15: process of retopography in 3DS Max.

Figure 16: Example of interactive PDF for William Macewen's Osteotome.

Figure 17: Module quiz format.

Figure 18: Home page of RCPSG online learning module.

\section{Biographical Notes}

Kirsty Earley- Digitisation Project Intern, Library and Heritage Department, Royal College of Physicians and Surgeons of Glasgow.

Dr. Daniel Livingstone- Programme Leader, School of Simulation and Visualisation, the Glasgow School of Art.

Dr. Paul Rea- Senior University Teacher, School of Life Sciences, College of Medical, Veterinary, and Life Sciences, University of Glasgow. 

